\title{
CFD study on laminar mixing at a very low Reynolds number by pitching and heaving a square cylinder
}

\author{
J. Ortega-Casanova \\ Universidad de Málaga, Andalucía Tech, Escuela de Ingenierías Industriales, \\ C/ Dr Ortiz Ramos s/n, 29071 Málaga, Spain \\ ${ }^{*}$ Corresponding author: jortega@uma.es \\ C.-H. Lai \\ University of Greenwich, UK \\ C.H.Lai@gre.ac.uk
}

\begin{abstract}
In this work, CFD is used to show how the combined motion of heaving and pitching a 2D square cyliner located in the middle of the channel can be used to enhance mixing of two fluids flowing at a very low Reynolds number along a rectangular channel. Both type of motioins are applied and enforced with a phase difference at the same time, but at difference frequencies and amplitudes, in order to see if mixing efficiency can be enhance. The characteristics of five governing input parameters and output results are examined. The input parameters consist of two frequencies, one for heaving and one for pitching, and two amplitudes, one for heaving and one for pitching. The output results are mixing efficiency, input power coefficient and mixing energy cost. The Optimal Space Filling algorithm is used to generate 60 different combinations of the input parameters which are used in the numerical studies. Different response surfaces of the output parameters are obtained. It is noted that certain combinations of the input parameters produce the highest mixing efficiency (around 55\%), the lowest input power coefficient (around 260 units). or the lowest mixing energy cost (around 13 units) are found. On the other hand a multi-objective optimisation is also carried out in order to find the input parameters that give rise to the highest possible mixing efficienty with lowest possible input power coefficient. A set of optimal solutions leading to Pareto Front is obtained which shows the trade-off between the objectives. Three potential optimal candidates are proposed in this paper.
\end{abstract}

Keywords: Micromixing, Rectangular cylinder, Low Reynolds numbers, Mixing efficiency, Mixing cost, Single and multi-objective optimisation. 


\section{Introduction}

Mixing with microscale is nowadays a great challenge due to the wide variety of LOC (Lab On a Chip) microdevices where an efficient mixing between entering fluids is highly desired. These devices are quite important specially in microfluidic systems, with applications for both biomedical and biological analysis, or for chemical synthesis. Some reviews on these topics are listed here [Beebe et al., 2002, Hessel et al., 2005, Mansur et al., 2008, Carpeto et al., 2011, Lee et al., 2011]. Due to the very small dimensions of the devices, the governing Reynolds number is quite small and laminar flow appears throughout the device. Together with frequently low molecular diffusion between mixing fluids, any kind of actions should be taken into account in order to improve mixing efficiency. One effective solution for enhancing efficiency is by increasing the interfacial contact surface between the different liquids to be mixed. Either active or passive mechanisms may be used and the aim is to reduce the diffusion distance using Fick's first law. There are many different ways that active mechanisms may be applied in order to increase the mixing efficiency. This paper examines such mechanism through the use of moving elements. Some related work in the literature, including the use of only the heaving motion of a square cylinder to improve mixing when fluids flow at a very low Reynolds number [Ortega-Casanova, 2016], the improvement of channel exit by simply pitching a square cylinder [Ortega-Casanova, 2017], and the development of a micromixer with a stirrer being drived at speeds of 100-600 rpm based on a micromachined magnetic-bar powered by a rotating magnetic field in some biological laboratory-on-a-chip applications [Lu et al., (2002)]. Lu et al in fact examined an array of bars and showed that at zero rotor spped the mxing efficiency was $80 \% 3 \mathrm{~mm}$ downstream, but when rotating at $600 \mathrm{rpm}$ the same efficiency was achieved as soon as the fluids passed the stirrer. This effect of enhancing mixing was confirmed at each of the rotor speeds they tested Ryu et al improved the micromixer that was proposed by Lu et al. [Lu et al., (2002)] and manufactured a "micro magnetic stir-bar integrated in parylene surfacemicromachined channels with improved design features, including small tolerance of the stir-bar to channel wall" [Ryu et al., (2004)]. This improved design increased not only the mixing efficiency but also showed that the magnetic stir-bar could be used to pump liquid along the microdevice. They also demonstrated that complete mixing was achieved instantly just downstream of the stirbar. In comparison with Lu et al., this full mixing was thought to be due to the fluid going through the stirrer instead of passing around it as happened in [Lu et al., (2002)]. Lin et al. presented a novel approach to achieve efficient mixing by using the Lorentz force to induce two counter-rotating 
streams producing a in-plane resonance of a rectangular microplate [Lin et al., (2011)]. After using different excitation voltages, Reynolds and Peclet numbers attained the maximum efficiency of around $93 \%$ at a Reynolds number of 0.0037 just one device length-scale downstream. Chen et al. studied a new beating behaviour of artificial cilia looking for rapid and complete fluid mixing in microdevices [Chen et al., 2014]. In comparison with the mixing with static cilia, for which the micromixing was relatively low, the beating of the artificial cilia made the mixing performance increased sharply up to around 90\%. Rahbar et al. also presented a micromixer Rah14 with artificial cilia doped with rare-earth magnetic powder which was controlled by magnetic fields. It was shown that the cilia can be manufactured as either individual microdevices or in arrays, which increase the mixing performance. An efficiency of around $85 \%$ in 3.5 minutes and that time could be reduced to 70 seconds by using an array of eight cilia filaments was obtained. Other recent studies dealt with different active mechanisms, among others, are those by Kunti et al. [Kunti et al., 2017] and Erkoc [Erkoç et al., 2016]. In the former study, a microgrooved channel floor with asymmetric pairs of electrodes and an array of them on the top wall consisting of a novel alternating current electrothermal micromixer, was able to obtain full, uniform and homogeneous mixing. In the latter study, a 2D T-jets mixer was used to assess the effect of the pulsation/modulation of jets flow rates on the dynamics of mixing. It was observed that the impact of the jets flow rate modulation on the flow field dynamics increases when modulation amplitude increases up to the point where it can completely drives the dynamics of the system.

As can be seen, different active mixing strategies can be used to enhance mixing where, when necessary and mainly by means of magnetic forces, certain parts of the microdevices can be forced to move which, usually, increases mixing efficiency. In this work the combined motions of heaving and pitching a 2D square cylinder located inside a straight channel is used to demonstrate the benefits when mixing two fluids at a low Reynolds and high Schmidt/Peclet numbers. This work can be see then as a third way of mixing fluids by means of moving a 2D square of which previous motions were heaving [Ortega-Casanova, 2016] and pitching [Ortega-Casanova, 2017] on its own. The aim of this work is to show optimal working points and as high as possible the mixing efficiency is as high as possible. Note that in single motion type of mixing the input power requirements and mixing energy cost are as low as possible. 


$$
\begin{aligned}
& \mathrm{l}[\mathrm{r}][\mathrm{r}][1] \hat{\ell} \mathrm{H}[\mathrm{c}][\mathrm{c}][1] \hat{H} \mathrm{xu}[\mathrm{c}][\mathrm{c}][1] \hat{X}_{u} \mathrm{xd}[\mathrm{c}][\mathrm{c}][1] \hat{X}_{d} \mathrm{~L}[\mathrm{c}][\mathrm{c}][1] \hat{L} \mathrm{x}[\mathrm{c}][\mathrm{c}][1] \hat{x} \mathrm{y}[\mathrm{c}][\mathrm{c}][1] \hat{y} 1 \mathrm{a}[\mathrm{c}][\mathrm{c}][0.9] \hat{U}, \rho, \mu \\
& 1 \mathrm{~b}[\mathrm{c}][\mathrm{c}][.9] \hat{m}=02 \mathrm{a}[\mathrm{c}][\mathrm{c}][.9] \hat{U}, \rho, \mu 2 \mathrm{~b}[\mathrm{c}][\mathrm{c}][.9] \hat{m}=\hat{m}_{o} 2[\mathrm{c}][\mathrm{c}][.8] 21[\mathrm{c}][\mathrm{c}][.8] 1 \mathrm{~h}[1][1][.75] \hat{h}(\hat{t}) \\
& \text { a[1][1][.75] } \alpha(\hat{t}) \text { Wall[r] [r] [.8] Wall Outlet[r][r][.8] Outlet }
\end{aligned}
$$

Figure 1: Sketch of the mixing domain.

\section{Geometry}

The proposed active mixing mechanism intends to mix two identical fluids entering through the left hand side of a 2D channel when the Reynolds number based on the channel width is very low.

Fig. 1 shows a sketch of the geometry in this study: it is $\hat{H}$ meters wide and $\hat{L}$ meters long $(\hat{-}$ is used to denote dimensional magnitudes). Both fluids enter with an identical and uniform velocity $\hat{U}$ and with the same physical properties such as, the density $\rho$, the viscosity $\mu$ and the mass diffusivity $D$ which are constants. The inlet streams have different solute mass concentrations. The lower one contains all solute (fluid 1) to be mixed while the upper one does not (fluid 2) (see Fig. 1). In the figure, one can also see the Cartesian coordinate system used throughout the study $(\hat{x}, \hat{y})$, with origin located in the middle of channel width and at a distance $\hat{X}_{u}$ downstream the inlet ( $\hat{x}$ is the streamwise direction while $\hat{y}$ the spanwise). The square cylinder, with side $\hat{\ell}$, is located initially at a distance from the cylinder centre to the side walls of $\hat{H} / 2$ and with an incidence angle of $45^{\circ}$, i.e. with its corners aligned with both coordinate axes. The position shown in Fig. 1 corresponds to a certain instant when the square cylinder has undergone heaving and pitching.

\section{The governing equations}

The equations governing the flow and mixing of both fluids are, as in [Ortega-Casanova, 2016, Ortega-Casanova, 2017], the unsteady Navier-Stokes (NS) equations (continuity and momentum equations) and the Advection-Diffusion (AD) equation. Therefore, under the consideration of an incompressible fluid with velocity $\hat{\mathbf{v}}$, pressure $\hat{p}$ and mass (concentration) of solute $\hat{m}$ of a specie moving with the fluid, the governing equations in a dimensionless way can be written as

$$
\begin{gathered}
\nabla \cdot \mathbf{v}=0 \\
\frac{\partial \mathbf{v}}{\partial t}+(\mathbf{v} \cdot \nabla) \mathbf{v}=-\nabla p+\frac{1}{R e} \nabla^{2} \mathbf{v} \\
\frac{\partial m}{\partial t}+(\mathbf{v} \cdot \nabla) m=\frac{1}{P e} \nabla^{2} m
\end{gathered}
$$


wall[c][c][1]Side wall 1[c][c][1]Sliding lines for heaving 2[c][c][1]Sliding line for pitching

Figure 2: Sketch of the sliding curves for Dynamic Mesh configuration.

where as characteristic magnitudes, as usual in channel flow problems, have been chosen $\hat{U}, \hat{H}$ and $\hat{H} / \hat{U}$ as characteristic distance, velocity and time, respectively, while regarding the mass of solute, $\hat{m}_{o}$ has been selected as its characteristic value ( $\rho \hat{U}^{2}$ would be the characteristic pressure). As can be seen from the set of governing equations (1)-(3), the dimensionless governing parameters are the Reynolds number $R e=\frac{\hat{U} \hat{H}}{\nu}$ and the Peclet number $P e=\frac{\hat{U} \hat{H}}{D}$, where $\nu$ is the kinematic viscosity and $D$ the mass diffusivity. Additionally, the Peclet number can be written in terms of the Reynolds number as $P e=R e S c$, where $S c$ is the Schmidt number given by $S c=\frac{\nu}{D}$, which is only fluid properties dependent.

This set of equations, together with the corresponding boundary conditions (see [Ortega-Casanova, 2016]), will be solved numerically in this work by means of the commercial software ANSYS-Fluent(C) [1] together with its dynamic mesh option to implement the pitching and heaving motions of the square cylinder. Fluent mathematical formulation was based on pressure, and all the discretization methods used for space and time were second order accuracy, while the velocity-pressure coupling was handled by the SIMPLE algorithm. The cylinder heaving and pitching oscillations were achieved by the Dynamic Mesh technique available in Fluent(C), as in [Ortega-Casanova, 2016, Ortega-Casanova, 2017], but now the mesh will slide around the sliding lines indicated in Fig. 2 to account for both kind of motions. Finally, the motion of the centre of gravity of the cylinder and its orientation are indicated by means of an User Defined Function (UDF in Fluent's terminology) where motion equations (see next Section) are implemented.

\section{Kinematics of the square cylinder}

With the square cylinder initially located at the origin of coordinates, as the time goes, it will heave with the spanwise location and velocity given, respectively, by

$$
h(t)=A_{h} \sin \left(2 \pi S t_{h} t\right)
$$

and

$$
V(t)=2 \pi S t_{h} A_{h} \cos \left(2 \pi S t_{h} t\right)
$$

where $A_{h}$ is maximum displacement of the pitching motion and $S t_{h}$ is the dimensionless frequency of the heaving oscillation, i.e. the heaving Strouhal number. The square cylinder will also pitch 
with the incidence angle and angular velocity given, respectively, by

$$
\alpha(t)=\alpha_{0}+A_{p} \sin \left(2 \pi S t_{p} t+\frac{\pi \phi}{180}\right)
$$

and

$$
\Omega(t)=2 \pi S t_{p} A_{p} \cos \left(2 \pi S t_{p} t+\frac{\pi \phi}{180}\right),
$$

where $\alpha_{0}$ and $A_{p}$ are the initial and maximum angle of the pitching motion, respectively, $S t_{p}$ is the dimensionless frequency of the pitching oscillation, i.e. the pitching Strouhal number, and $\phi$ is, in degrees, the phase difference between the heaving and pitching motions.

Initially, at $t=0, \alpha=\alpha_{0}\left(=45^{\circ}\right)$ and $h=0$, being the upper (or lower) initial gap between

the cylinder and the channel walls given by $H_{g}=\frac{H-2 \ell \sin (\pi / 4)}{2}$. Regarding the amplitudes $A_{h}$ and $A_{p}$, they will be rewritten as $A_{h}=K_{h} H_{g}$ and $A_{p}=K_{p} 2 \pi$, where $K_{h}$ is the percentage, so much per one, of $H_{g}$ the cylinder moves up and downward while $K_{p}$ is also the percentage, so much per one, of a complete revolution. The range of both motion amplitudes used in this study are $0.1 \leq K_{h} \leq 0.8$ and $0.05 \leq K_{p} \leq 1$

\section{Numerical considerations}

Both in [Ortega-Casanova, 2016] and [Ortega-Casanova, 2017] were carried out validation tests to assess if the followed methodology was good enough to solve both low Reynolds number flows around square cylinders and mixing fluid problems. It was then shown that both kind of problems were solved adequately by means of Fluent software. Additionally, grid and time step convergence studies were also done in order to find the optimal mesh size $d s$ and time step $\Delta t$. For that reason, the same optimal configurations used in [Ortega-Casanova, 2016, Ortega-Casanova, 2017] have been used now, since they were shown to give very good results. In particular, regarding the time step, it has been now based on the highest frequency motion (lowest oscillation period) thus $S t_{M}=\max \left(S t_{h}, S t_{p}\right)$ so $\Delta t=S t_{M}^{-1} / N$, with $N=2000$ being the time steps per lowest oscillation period. Regarding the size $d s$ of the used mesh, $d s / \ell=0.025$ with a discretization uncertainty in terms of GCI of $\sim 2.41 \%$.

\section{Assessment of the mixing}

To assess the quality of a device in mixing fluid processes, it is frequently used a mixing efficiency. It is based on the mass fraction standard deviation $\sigma$ of one of the fluid at the channel 
outlet. Thus, by using the standard deviation $\sigma$ given by

$$
\sigma(t)=\left\{\int_{-1 / 2}^{1 / 2}\left[m\left(t, y^{\prime}\right)-\bar{m}\right]^{2} d y^{\prime}\right\}^{1 / 2},
$$

the mixing efficiency $\eta$, in \%, is then defined as [Ortega-Casanova, 2016, Cheri et al., 2013, Hsiao et al., 2014, Sarkar et al., 2014]

$$
\eta(t)=\left(1-\frac{\sigma(t)}{\sigma_{\max }}\right) \times 100 .
$$

The magnitude $\bar{m}$ in (8) is the mean mass fraction at the channel outlet while in $(9), \sigma_{\max }$ is the maximum standard deviation at inlet (in our problem, both $\bar{m}$ and $\sigma_{\max }$ are 0.5). Besides this, since $0 \leq m \leq 1$, at the outlet we will have $0 \leq \sigma \leq 0.5$ : $\sigma=0$ means full mixing $(\eta=100 \%)$; and $\sigma=0.5$ no mixing at all $(\eta=0 \%)$.

In [Ortega-Casanova, 2016], a mixing energy cost mec was also used to identify the best combination of input parameters in order to find out the heaving configuration giving the best mixing but with the lowest input power requirements, i.e. the cheapest configuration from a cost point of view, so it will be also used now. To that end, first of all, the input power requirements to run the channel and pitch and heave the square cylinder must be assessed. In terms of input power coefficients (made dimensionless, as usually, with $\frac{1}{2} \rho \hat{U}^{3} \hat{H}$ ), firstly, the one for running the channel can be evaluated as [Ortega-Casanova, 2016, White, 2003]

$$
C_{P_{\text {running }}}(t)=2 E u(t),
$$

with $E u(t)=\Delta \hat{p}(t) /\left(\rho \hat{U}^{2}\right)$ being the instantaneous Euler number based on the pressure drop along the channel (in (10) it has also been taken into account that dimensionless flow rate is unity). Secondly, the input power coefficient for pitching the square cylinder can be evaluated as [Ortega-Casanova, 2017, Ashraf et at., 2011, Hoke et al., 2015]

$$
C_{P_{\text {pitching }}}(t)=-C_{M}(t) \Omega(t) \ell^{2}
$$

with $C_{M}=\hat{M} /\left(\frac{1}{2} \rho \hat{U}^{2} \hat{\ell}^{2}\right)$ being the coefficient of moment and $\hat{M}$ the pitching moment. And thirdly, the corresponding input power needed to heave the cylinder, in terms of the corresponding input power coefficient, can be written as [Ortega-Casanova, 2016]

$$
C_{P_{\text {heaving }}}(t)=-\ell C l(t) V(t)
$$

with $C l(t)$ being the instantaneous lift coefficient. The way input power coefficients have been made dimensionless is the reason because $\ell$ appears in (12), since $C l$ has been based on $\hat{\ell}$. 


\section{$\operatorname{Sth}[\mathrm{c}][\mathrm{c}][0.5] S t_{h} \operatorname{Stp}[\mathrm{c}][\mathrm{c}][0.5] S t_{p} \mathrm{Kh}[\mathrm{c}][\mathrm{c}][0.5] K_{h} \mathrm{Kp}[\mathrm{c}][\mathrm{c}][0.5] K_{p} \operatorname{desf}[\mathrm{c}][\mathrm{c}][0.5] \phi$}

$(6)$

ISSP

LST

ppphimate

Figure 3: Input parameter planes with the corresponding simulated values.

Now, if all three input power coefficients are integrated together in time over a period $T$, its time-averaged value can be evaluated as

$$
\overline{C_{P}}=\frac{1}{T} \int_{t_{0}}^{t_{0}+T}\left(C_{P_{\text {running }}}\left(t^{\prime}\right)+C_{P_{\text {pitching }}}\left(t^{\prime}\right)+C_{P_{\text {heaving }}}\left(t^{\prime}\right)\right) d t^{\prime} .
$$

$t_{0}$ is a reference time once the flow reaches a periodic, or quasi-periodic, behaviour. Similar expressions could be used to get mean input running, pitching and heaving coefficients). Finally, the mixing energy cost can be evaluated as [Ortega-Casanova, 2016]

$$
\operatorname{mec}=\overline{C_{P}} / \bar{\eta},
$$

where, due to the fact that $\eta$ is time-dependent, its time-averaged value $\bar{\eta}$, calculated as

$$
\bar{\eta}=\frac{1}{T} \int_{t_{0}}^{t_{0}+T} \eta\left(t^{\prime}\right) d t^{\prime}
$$

will be also used.

To assess how good pitching and heaving motions are when are used to mix fluids at low Reynolds numbers, $\bar{\eta}, \overline{C_{P}}$, and $m e c$ will be evaluated in next Section 7 .

\section{Results}

Once the optimal mesh and number of time steps per lowest oscillation period were chosen, several numerical simulations have been conducted for different values of the 5 input parameters while, as in [Ortega-Casanova, 2016, Ortega-Casanova, 2017], the Reynolds and Peclet/Schmidt numbers have been fixed in the study: $R e=1, P e=S c=10^{4}$. Additionally, instead of study a big range of input parameter potential good values, we have focus on a small range around their optimal values found in [Ortega-Casanova, 2016, Ortega-Casanova, 2017]. In particular, $0.5 \leq$ $S t_{h}, S t_{p} \leq 1.5,0.1 \leq K_{h} \leq 0.8,0.05 \leq K_{p} \leq 1$ and $0^{\circ} \leq \phi \leq 360^{\circ}$. To select their corresponding studied values we made use of the ANSYS Design of Experiments (DOE) technique, which allows 


\begin{tabular}{c|ccccc||ccc} 
& \multicolumn{5}{|c||}{ Input parameters } & \multicolumn{3}{c}{ Output parameters } \\
\cline { 2 - 8 } & $K_{h}$ & $K_{p}$ & $S t_{h}$ & $S t_{p}$ & $\phi$ & $\bar{\eta}[\%$ & $\overline{C_{p}}$ & mec \\
\hline $\min (\bar{\eta})$ & 0.176 & 0.108 & 1.342 & 0.942 & $207^{\circ}$ & $\mathbf{7 . 0}$ & 531.0 & 75.3 \\
$\min \left(\overline{C_{p}}\right)$ & 0.561 & 0.042 & 0.658 & 0.975 & $261^{\circ}$ & 24.5 & $\mathbf{4 9 6 . 6}$ & 20.2 \\
$\min (m e c)$ & 0.771 & 0.242 & 0.908 & 0.692 & $99^{\circ}$ & 28.6 & 537.3 & $\mathbf{1 8 . 7 7}$ \\
\hline $\max (\bar{\eta})$ & 0.712 & 0.958 & 1.158 & 0.875 & $117^{\circ}$ & $\mathbf{4 8 . 3}$ & 1714.8 & 35.5 \\
$\max \left(\overline{C_{p}}\right)$ & 0.631 & 0.892 & 0.992 & 1.442 & $225^{\circ}$ & 42.0 & $\mathbf{3 2 4 5 . 0}$ & 77.3 \\
$\max (m e c)$ & 0.631 & 0.892 & 0.992 & 1.442 & $225^{\circ}$ & 42.0 & 3245.0 & $\mathbf{7 7 . 3}$
\end{tabular}

Table 1: Remarkable results from the 60 simulated cases.

the user to conduct a series of experiment with a given set of parameters, which range between known values, in order to minimize the number of runs to understand how the parameters affect the problem solution. Thus, the Optimal Space Filling (OSF) algorithm [Biancoline et al., 2014], which chooses the design points avoiding having samples with same values of any of the input parameters [?, more details about the algorithm can be consulted by the reader in]]ansdoe is used to generate 60 numerical experiments for different values of the input parameters. Their corresponding values, in different input parameter planes, can be seen in Fig. 3.

\subsection{Remarkable simulated results}

Once all simulations have been carried out and ready to be analysed, in this Section only some remarkable cases of the simulated ones will be presented and discussed. From the 60 cases, only 6 have been considered as remarkable and they are those giving the highest and lowest values of output parameters. In that sense, Table 1 summarises the input parameters of the simulated configurations with maximum and minimum values of efficiency $\bar{\eta}$, input power coefficient $\overline{C_{p}}$ and mixing energy cost mec. Regarding the efficiency, as can be seen, its minimum value takes place at low motion amplitudes, both for pitching and heaving, for heaving Strouhal numbers above unity while for pitching Strouhal numbers below unity. On the other hand, the maximum efficiency is reached for almost the highest motion amplitudes and Strouhal numbers around unity, above one for heaving and below one for pitching. Regarding $\overline{C_{P}}$, its maximum and minimum values take place for $K_{h} \sim 0.6$, while the minimum for nearly null pitching oscillation amplitude, $K_{p} \sim 0$, and the maximum for a high values of $K_{p}$. This indicates the strong effect of the pitching amplitude on the global input power energy needed to generate the combined pitching and heaving motions. 
$($ (a)

$\eta$ ALEass

$7480 \%$

tion

col-

ormap

Figure 4: Mass fraction contours for input parameters giving: (a) $\min (\bar{\eta})$; and (b) $\max (\bar{\eta})$. (c) is the colormap to read contour colour levels.

$$
\mathrm{t}[\mathrm{c}][\mathrm{c}][0.7] \text { tefficiency[c] }][\mathrm{c}][0.7] \eta \text { eta } \max [1][1][0.5] \max (\bar{\eta}) \text { eta } \min [1][1][0.5] \min (\bar{\eta})
$$

Figure 5: Temporal evolution of mixing efficiency at device exit.

Finally, the minimum mec value takes place for low and high values of the pitching and heaving amplitudes, respectively, while the maximum mec occurs at medium-high amplitudes for both pitching and heaving amplitudes. It must be noted that, from the 60 simulations, the ones with $\max \left(\overline{C_{P}}\right)$ and $\max (m e c)$ are the same.

In order to have an idea about how the worst and best efficiency configurations look like, Fig. 4 shows solute mass fraction contours on the whole domain. As can be seen, in the lowest efficiency configuration, the low pitching and heaving motion amplitudes hardly perturb the interface between the mixing fluids, which gives place to an almost flat and unperturbed contact surface. On the other hand, Fig. 4(b) clearly shows why this configuration is the best one: the combined pitching and heaving motions create a specific and quite irregular pattern downstream with high contact interface, and thus interaction, between the mixing fluids. As one could expect, the flow field is highly time dependent and this can be observed in the temporal evolutions of the efficiency shown in Fig. 5, where those for the configuration giving maximum and minimum efficiency are shown. After certain dimensionless time, both evolutions reach a quasi-periodic evolution, being quicker to reach that time the lowest efficiency configuration than the highest one.

\subsection{Response surfaces}

In order to carry out future optimisation tasks, the Response Surface Methodology [Myers et al., 2016] will be used by means of the 60 different numerical results corresponding to the previously commented simulated cases. Once the response surfaces are generated, they will be used later to find 


\begin{tabular}{c|ccc}
\multirow{2}{*}{ Goodness of fit } & \multicolumn{3}{|c}{ Response surface } \\
\cline { 2 - 4 } & $\eta$ & $\overline{C_{p}}$ & mec \\
\hline Root Mean Square error & $10^{-7}$ & $7.8 \times 10^{-5}$ & $7.7 \times 10^{-6}$ \\
Coefficient of Determination $R^{2}$ & 1 & 1 & 1
\end{tabular}

Table 2: Goodness of fitting the indicated output parameters with response surfaces.

optimal combinations of input parameters giving best values of any output parameter. Therefore, a response surface for each output parameter will be obtained but, due to the fact that there are 5 input parameters, to visualize some of the surfaces we are going to fix three input parameters so a surface can be obtained as other two input parameters change in their studied range. The goodness of the response surfaces to fit all simulated output parameters can be assessed by different indexes. In our case, the Root Mean Square Error between the response surface predictions and the simulated values, and the Coefficient of Determination $R^{2}$ have been evaluated and shown in Table 2. As can be seen, both indexes indicate a very good prediction of the response surface in relation to the simulated results of the corresponding output parameters. With this in mind and as examples, different response surfaces can be seen in Fig. 6 for $\bar{\eta}, \overline{C_{p}}$ and mec when specific values for $K_{h}, K_{p}$ and $\phi$ have been used. Actually, $\phi$ has been fixed to its medium value, i.e. $\phi=180^{\circ}$, while other two corresponding to pitching and heaving amplitudes have changed. Firstly, Fig. 6(a)-(c) are results for low values of motion amplitudes $\left(K_{h}=0.1, K_{p}=0.05\right)$. They show that the efficiency is quite low, between 10 and 20\%, with the minimum efficiency taking place at unity Strouhal numbers, i.e. $S t_{h} \sim S t_{p} \sim 1$. It is also around these specific Strouhal numbers where, on the one hand, the input power coefficient has its minimum and, on the other, the mixing cost its maximum. Secondly, Fig. 6(d)-(f) corresponds to medium values of motion amplitudes $\left(K_{h}=0.45, K_{p}=0.475\right)$. In these figures one can observe that: the efficiency increases with its minimum values moving to the right-down side of the plane, i.e. high $S t_{h}$ and low $S t_{p}$ values, and a clear maximum of the efficiency appears at $S t_{h} \sim 0.75$ and $S t_{p} \sim 1.25$; the low region of input power coefficient moves to low values of $S t_{p}$, while high values of input power coefficient appears for high values of $S t_{p}$ with $S t_{h} \sim 1$ in both cases; and that the cheaper configurations, from a mixing energy cost point of view, are those with low Strouhal number values $(\sim 0.7)$ while the more expensive ones are those with the highest $S t_{p}$ and medium-high values of $S t_{h}$. And thirdly, Fig. $6(\mathrm{~g})$-(i) are for highest used values of motion amplitudes $\left(K_{h}=0.8, K_{p}=1\right)$. As can be seen, it 
Figure 6: (a)-(c) for $K_{h}=0.1, K_{p}=0.05, \phi=180^{\circ}$; (d)-(f); for $K_{h}=0.45, K_{p}=0.475, \phi=180^{\circ}$; and (g)-(i) for $K_{h}=0.8, K_{p}=1, \phi=180^{\circ}$.

is observed that the maximum efficiency of $\sim 45 \%$ moves to unity values of Strouhal numbers; in comparison with previous motion amplitudes, the input power coefficient increases according to the increase in the motion amplitudes and the maximum values region appears, again, for medium $S t_{h}$ and high $S t_{p}$ values; and that the cheapest configurations are now those with medium $S t_{h}$ and low $S t_{p}$ values, being the cost of maximum efficiency configuration around 40 units for high $S t_{p}$ values and $S t_{h} \sim 1$.

Obviously, this analysis has been done for certain values of motion amplitudes and phase difference, which means that by using other values the behaviour and response surfaces could be different. As mentioned previously, this is only one example of different response surfaces obtained from the simulated results. The main interest of this kind of surfaces is that they can be used to carry out optimisation tasks, both single- and multi-objective, in order to find combinations of input parameters which gives maximum or minimum values of output parameters, as it will be shown in next two sections.

\subsection{Single-objective optimisation}

In this Section, the Response Surface Methodology will be used to get optimal values of the efficiency $\bar{\eta}$, the input power coefficient $\overline{C_{p}}$ and the mixing energy cost mec, which correspond with their absolute maximum, minimum and minimum value, respectively. Table 3 summarises these optimal values together with the input parameter configurations to get them. Regarding the maximum mixing efficiency, from the first row of Table 3 one can see that it takes place for around unity values of Strouhal numbers (around the same than the ones shown in Fig. 6(g)), high pitching and medium heaving amplitudes and medium values of phase difference between both motions. From a cost point of view, this optimal efficiency configuration with mec $\simeq 50$ is not one of the more expensive as Fig. 6(i) shows, with other configurations with mec $\sim 65$. Regarding the minimum input power coefficient, from the second row of Table 3 one can see that it takes place for Strouhal numbers a little smaller than unity, with low pitching and medium 


\begin{tabular}{c|ccccc||ccc} 
& \multicolumn{5}{|c||}{ Input parameters } & \multicolumn{3}{c}{ Output parameters } \\
\cline { 2 - 8 } & $K_{h}$ & $K_{p}$ & $S t_{h}$ & $S t_{p}$ & $\phi$ & $\bar{\eta}[\%]$ & $\overline{C_{p}}$ & mec \\
\hline $\max (\bar{\eta})$ & 0.600 & 0.909 & 1.139 & 1.080 & $163.057^{\circ}$ & $\mathbf{5 3 . 5}$ & 2549.9 & 49.8 \\
$\min \left(\overline{C_{p}}\right)$ & 0.471 & 0.173 & 0.972 & 0.868 & $175.933^{\circ}$ & 13.0 & $\mathbf{2 6 0 . 4}$ & 20.0 \\
$\min ($ mec $)$ & 0.680 & 0.226 & 0.802 & 0.909 & $177.993^{\circ}$ & 27.6 & 367.61 & $\mathbf{1 3 . 3}$
\end{tabular}

Table 3: Summary of input parameters for optimal $\bar{\eta}, \overline{C_{p}}$ and mec configurations. The corresponding output parameters are also indicated.

heaving amplitudes and, again, medium values of the phase difference. The main inconvenient of this low power coefficient is the low efficiency it gives with $\bar{\eta} \simeq 13 \%$. Due to the low input power coefficient, the cost of this configuration is also low, but is not the lowest one. And regarding the minimum mixing energy cost, from table third row one can see that it takes place, again, for Strouhal numbers a little smaller than unity, amplitudes a little higher than those for minimum power coefficient configuration (second row of the table) and, as in the two other optimal cases, for medium values of phase difference. The fact that the motion amplitudes are a little higher means, in comparison with $\min \left(\overline{C_{p}}\right)$ configuration, that efficiency is doubled while the power coefficient increases only $\sim 1.4$ times, which gives as a result a lower, actually the lowest, mixing energy cost.

As can be seen in Table 3, the best mixing efficiency configuration gives bad both input power coefficient and mixing energy cost. This is the problem when optimising just one objective, but other optimisation techniques can be applied to optimise more than one objective and then we have which is called as multi-objective optimisation, as the reader will see in next Section.

\subsection{Multi-objective optimisation}

This section is devoted to find the optimal input parameters combinations to optimise not one objective, as in the previous section, but two of them by means of a multi-objective optimisation technique. In our case, the optimisation process has been conducted by using multi-objective genetic algorithms. They will allow us to to find certain combinations of the input parameters which lead to the best values of the corresponding selected objectives. A Genetic Algorithm (GA) is one of the most employed global optimisation techniques. It, briefly, consists in a stochastic optimisation method based on the evolutionary theory proposed by Darwin and also on the survival of the fittest scheme [Goldberg, 1989]. Additionally, it takes into account that the best solutions will be found in zones of the input parameter space where good solutions are able to be found. 


\begin{tabular}{|c|c|c|c|c|c|c|c|c|}
\hline \multirow[b]{2}{*}{ POD \# } & \multicolumn{5}{|c|}{ Input parameters } & \multicolumn{3}{|c|}{ Output parameters } \\
\hline & $K_{h}$ & $K_{p}$ & $S t_{h}$ & $S t_{p}$ & $\phi$ & $\bar{\eta}[\%]$ & $\overline{C_{p}}$ & mec \\
\hline $\mathrm{A}$ & 0.647 & 0.938 & 1.139 & 1.000 & $153.270^{\circ}$ & 52.75 & 2229.2 & 42.26 \\
\hline B & 0.738 & 0.538 & 0.812 & 0.904 & $217.872^{\circ}$ & 35.69 & 897.0 & 25.13 \\
\hline $\mathrm{C}$ & 0.505 & 0.183 & 0.924 & 0.867 & $189.294^{\circ}$ & 16.19 & 264.1 & 16.31 \\
\hline
\end{tabular}

Table 4: Summary of input parameters for indicated PODs. The corresponding output parameters are also included.

Therefore, those regions are exhaustively explored by searching from a set of design points (also called population). These points are later manipulated by means of genetic operators to obtain a new population of input parameters. These processes are repeated till a certain percentage of the population converges to the same design. When a certain GA is used for multi-objective optimisation purposes, the Multi-Objective Genetic Algorithm (MOGA) usually converges to a population which consists of individuals belonging to a certain curve known as the Pareto Front $(\mathrm{PF})$ of the solutions. However, the use of MOGA needs a high number of evaluations of the objective functions in order to reach an optimum configuration. This will be avoided in the optimisation methodology we are going to apply by using response surfaces, as the ones shown in Section 7.2 , that can be obtained from the 60 simulations to evaluate the objective functions at the needed input parameters. Besides this, it also avoids new and expensive CFD simulations of some thousands of input parameter combinations which are required to get the final set of optimum solutions.

One can find in the literature different multi-objective genetic algorithms [?, e.g., see]among others]Deb01. However, the Non-dominated Sorting Genetic Algorithm (NSGA-II) [Deb et al., 2000] and the Strength Pareto Evolutionary Algorithm 2 (SPEA2) [Zitzler et al., 2001] are the two mains and more used ones when multi-objective optimisation is required. Despite NSGA-II and SPEA2 algorithms are quite similar both in characteristics and performances, the NSGA-II is the one used due to the fact its effectiveness is higher when finding the Pareto optimum solutions, see [Ortega-Casanova and Castillo-Sanchez, 2017] for another work where it has been used.

To start with, let's suppose we are interested in finding input parameter combinations giving the maximum possible value of the mixing efficiency $\bar{\eta}$ with the minimum possible input power coefficient $\overline{C_{P}}$. After the NSGA-II algorithm is run, the PF with the obtained set of optimal solutions is obtained and it can be seen as solid squares in Fig. 7. It has been also included a 
eta $[\mathrm{c}][\mathrm{c}][0.5] \bar{\eta} \mathrm{Cp}[\mathrm{c}][\mathrm{c}][0.5] \overline{C_{P}} \mathrm{~A}[\mathrm{c}][\mathrm{c}][0.5] \mathrm{AB}[\mathrm{c}][\mathrm{c}][0.5] \mathrm{BC}[\mathrm{c}][\mathrm{c}][0.5]$ Cpareto front[1][1][0.45]Pareto

Frontspline fitting[1][1][0.45]Spline fittingdesign points[1][1][0.45]PODs

Figure 7: Pareto Front with the set of optimal solutions with high mixing efficiency and low input power coefficient. The figure also includes the spline fitting $\left(R^{2} \simeq 0.99\right)$ and three PODs.

non-linear least square fitting of the corresponding points indicated by a solid curve. As one can see, an improvement in one objective (higher values of $\bar{\eta}$ ) means a degradation of the other (higher values of $\overline{C_{P}}$ ) and vice-versa, i.e. it shows a clear trade-off between the mixing efficiency and the input power coefficient: an increase in $\bar{\eta}$ gives as result an increase $\overline{C_{P}}$. Additionally, the PF also indicates that the nature of this trade-off is not uniform with optimal solution with both high or low efficiency. To analyse the Pareto-optimal set of solutions regarding the performance of the two objectives, three representative Pareto-Optimal Designs (PODs) have been selected. They are indicated in Fig. 8 with solid circles and letters A, B and C and summarised in Table 4. These PODs have been selected from the PF candidates but having high, medium and low efficiency, i.e. POD A, B and C, respectively. However, other user may use his/her preference to select other representative PODs that can help to explain the trend in Pareto-optimal set. In particular, PODs $\mathrm{A}$ and $\mathrm{C}$ are close to the extreme ends of the PF, as shown in Fig. 8, indicating $\bar{\eta}$-oriented and $\overline{C_{P}}$-oriented designs, respectively: POD A will give high $\bar{\eta}$ with the high $\overline{C_{P}}$, while POD C will give low $\bar{\eta}$ with low $\overline{C_{P}}$. On the contrary, POD B will give an intermediate solution between $\mathrm{A}$ and $\mathrm{C}$ with medium values of both $\bar{\eta}$ and $\overline{C_{P}}$.

Once the set of optimal solutions is drawn as a PF of the optimised output parameters, $\bar{\eta}$ and $\overline{C_{P}}$, another PF can be plotted with optimal values of the remaining output and input parameters. This can be seen in Fig. 8 where 6 more PFs are depicted: the PF is indicated with solid squares, the non-linear least square fitting with a solid curve and the PODs with solid circles. In particular, subfigure (a) shows the $\bar{\eta}-$ mec PF which can help the end user to know the mixing energy cost for a desired efficiency of the PF. From this PF, one can even obtain the efficiency of the cheapest (lowest mec) configuration, which corresponds to mec $\simeq 13$ and $\bar{\eta} \sim 25 \%$, which nearly coincides with the absolute $\min (m e c)$ configuration shown in Table 3. Subfigure (b) shows the $\bar{\eta}-K_{h} \mathrm{PF}$ which gives the optimal values of $K_{h}$ for $\bar{\eta}$ values on the optimal set. As can be seen, the highest efficiencies are obtained for $0.6 \lesssim K_{h} \lesssim 0.7$ and it is also noticeable that for $\bar{\eta} \lesssim 30, K_{h}$ almost changes linearly. Subfigure (c) shows the $\bar{\eta}-K_{p}$ PF where clearly can be seen well defined values for low efficiencies, for which $K_{p} \sim 0.2$, and high efficiencies, for which $K_{p} \sim 0.9$. Regarding the 
$\operatorname{eta}[\mathrm{c}][\mathrm{c}][0.5] \bar{\eta} \operatorname{mec}[\mathrm{c}][\mathrm{c}][0.5]$ mecSth$\left._{\mathrm{c}}\right][\mathrm{c}][0.5] S t_{h} \mathrm{Stp}[\mathrm{c}][\mathrm{c}][0.5] S t_{p} \mathrm{Kh}[\mathrm{c}][\mathrm{c}][0.5] K_{h} \mathrm{Kp}[\mathrm{c}][\mathrm{c}][0.5] K_{p}$ pareto front[1][1][0.4]PFspline fitting[1][1][0.4]Spline fit.design points[1][1][0.4]PODs

(a) $(6)$

HAt2

009090886

Figure 8: Pareto Fronts for the indicated parameters. The Coefficient of Determination $R^{2}$ is also indicated for each PF.

Strouhal numbers, on the one hand, subfigure (d) shows the $\bar{\eta}-S t_{h}$ PF which gives the optimal values of $S t_{h}$ for $\bar{\eta}$ on the optimal set. One can see that $S t_{h}$ values above unity usually gives high mixing efficiencies with its highest values for $1.1 \lesssim S t_{h} \lesssim 1.2$. On the other, subfigure (e) shows the the $\bar{\eta}-S t_{p}$ PF which gives the optimal values of $S t_{p}$ for $\bar{\eta}$ on the optimal set. One can see that

there is a range of optimal efficiencies with $15 \lesssim \bar{\eta} \lesssim 40 \%$ which takes place for $S t_{p} \sim 0.9$ but $S t_{p}$ needs to be around unity to get the highest efficiency of $\sim 55 \%$. Finally, subfigure (f) shows the $\bar{\eta}-\phi$ PF which gives the optimal values of $\phi$ for $\bar{\eta}$ values on the optimal set. Three different regions are clearly observed: one for low efficiencies, with $\phi \sim 170^{\circ}$; another for medium efficiencies, with $\phi \sim 220^{\circ}$; and the third one for high efficiencies, with $\phi \sim 150^{\circ}$.

As it has been shown, when dealing with multi-objective optimisation (two objectives in our case), genetic algorithms are a good option to carry out the optimisation process. Once it finishes, the PF with the set of optimal solutions can be obtained. This set of solutions represents a tradeoff between the desired objectives and none of these Pareto-optimal solutions is superior to the other ones for both objectives. Thus, the final choice by the user is important when selecting a solution of the PF that meets his/her needs. In that sense, three optimal solutions from the PF have been proposed (PODs \#A, \#B or \#C). However, the final application can help to decide if it is better to have higher values of one objective and lower ones of the other or vice versa.

\subsection{Comparison with previous works}

In this section we are going to compare the present results with previous ones in order to have an idea about how good the present active mixing mechanism is, in terms of efficiency, power input energy and mixing energy cost, in comparison with other mechanisms both active and passive.

Table 5 summarised output mixing results from the present work and 7 more for configurations giving the highest mixing efficiency at the indicated Reynolds number. First of all, it is interesting 


\begin{tabular}{c|c|c|c|c|c|c|c|c} 
& \multicolumn{7}{|c}{ Author, Re } \\
\cline { 2 - 9 } & Present, 1 & Ort16, 1 & Ort17, 1 & Chu07, 1 & Che13, 1 & Hsi14, 1 & Par14, 10 & Ort18, 0.1 \\
\hline$\overline{C_{P}} \simeq$ & 2550 & 1000 & 2500 & $10^{4}$ & 120 & $9 \times 10^{3}$ & 795 & $33 \times 10^{4}$ \\
\hline $\bar{\eta} \simeq$ & 54 & 30 & 46 & 55 & 60 & 70 & 40 & 95 \\
\hline$m e c \simeq$ & 50 & 33 & 56 & 182 & 2 & 128 & 20 & 350 \\
\hline
\end{tabular}

Table 5: Comparison of mixing parameters got by different authors.

to make a comparison with heaving [Ortega-Casanova, 2016] and pitching [Ortega-Casanova, 2017] mechanisms working separately at the same Reynolds and Schmidt numbers when the highest efficiency is wanted. As can be seen, in the present combined mechanism, the input power coefficient is the highest one, 2.5 times higher than when just heaving and only few units higher than when just pitching. This clearly shows that, to get highest efficiencies, the pitching motion is a highly energy-consumed motion. On the other hand, the mixing efficiency got in the present work is also higher than when just heaving or pitching, being $80 \%$ and $17 \%$ higher, respectively. However, regarding the cost of getting just $1 \%$ of efficiency, despite the fact the present combined mixing mechanism has the highest input power coefficient, it is not the one with the highest cost since the highest mixing efficiency it gives makes mec be a little lower than when just pitching, although it is higher than when just heaving. Additionally, it has been also included in Table 5 other works with mixing studies at the same Reynolds number when using not active but passive mixing mechanisms. In particular, in the work by Chu07, similar mixing efficiencies than here are obtained but with a higher cost. However, in works by Che13 and [Hsiao et al., 2014], higher mixing efficiencies are reported with much lower and much higher mixing energy cost, respectively. In that sense, it is remarkable the passive mixing mechanism presented in [Cheri et al., 2013], where geometries with expansion chambers and obstacles inside them were studied with quite satisfactorily results both from mixing efficiency and input power energy points of view. Finally, regarding different Reynolds numbers, Par14 were able, for a higher Reynolds number, to get mixing with lower cost but also with lower efficiency, while Ort18 obtained, for a lower Reynolds number, a much expensive mixing but with an almost full mixing. This shows that, frequently, high values of mixing efficiency need high requirements of input power energy to get efficient mixing, so multi-objective optimisation, as it has been carried out in this work, is a good option to find a trade-off between the high efficiency and low input energy needs. 


\section{Conclusions}

CFD has been used to assess the mixing performance of a mixing device based on the combined motion of both heaving and pitching a 2D square cylinder located inside a straight channel. Thanks to a total of 60 numerical simulations for different values of the 5 governing input parameters (i.e.

pitching and heaving frequencies, amplitudes and phase difference), different response surfaces of the 3 output parameters (i.e. mixing efficiency, input power coefficient and mixing energy cost) have been able to be obtained. From them, it has been found optimal combinations of the input parameters in order to get: the highest mixing efficiency of $\sim 54 \%$; the lowest input power coefficient of $\sim 260$ units; and the lowest mixing energy cost of $\sim 13$ units. Regarding the combined motion giving the highest mixing efficiency, it is worth saying that the highest efficiency is higher than when mixing by heaving (with $\bar{\eta} \sim 30 \%$ ) or pitching (with $\bar{\eta} \sim 46 \%$ ) separately, being remarkable that the mixing energy cost is now even lower $(\sim 50)$ than when just pitching (for which mec $\sim 56$ ) because of the higher efficiency of the combined motion. It has been also carried out a multiobjective optimisation looking for input parameter combinations giving optimal solutions with high efficiency and low input power coefficient. In this case, a set of optimal solutions is proposed from which the end user can choose any of them depending on the desired mixing efficiency and power input coefficient: high efficiency solutions means high input power needs and vice versa. Once both the mixing efficiency and power input are chosen from the optimal set, the end user can also estimate the input parameters giving the desired optimal results. Just as an example, three optimal candidates are proposed: with high, medium and low mixing efficiency.

\section{References}

[Ortega-Casanova, 2016] Ortega-Casanova, J. (2016). Enhancing mixing at a very low Reynolds number by a heaving square cylinder. Journal of Fluids and Structures, 65, 1-20.

[Ortega-Casanova, 2017] Ortega-Casanova, J. (2017). CFD study on mixing enhancement in a channel at a low reynolds number by pitching a square cylinder. Computers \& Fluids, 145, 141-152.

[Beebe et al., 2002] Beebe, D. J., Mensing, G. A., \& Walker, G. M. (2002). Physics and applications of microfluidics in biology. Annual Review of Biomedical Engineering, 4(1), 261-286. 
[Hessel et al., 2005] Hessel, V., Löwe, H., \& Schönfeld, F. (2005). Micromixers-a review on passive and active mixing principles. Chemical Engineering Science, 60(8), 2479-2501.

[Mansur et al., 2008] Mansur, E. A., Ye, M., Wang, Y., \& Dai, Y. (2008). A state-of-the-art review of mixing in microfluidic mixers. Chinese Journal of Chemical Engineering, 16(4), 503-516.

[Carpeto et al., 2011] Capretto, L., Cheng, W., Hill, M., \& Zhang, X. (2011). Micromixing within microfluidic devices. In Microfluidics (pp. 27-68). Springer Berlin Heidelberg.

[Lee et al., 2011] Lee, C. Y., Chang, C. L., Wang, Y. N., \& Fu, L. M. (2011). Microfluidic mixing: a review. International Journal of Molecular Sciences, 12(5), 3263-3287.

[Lu et al., (2002)] Lu, L. H., Ryu, K. S., \& Liu, C. (2002). A magnetic microstirrer and array for microfluidic mixing. Microelectromechanical Systems, Journal of, 11(5), 462-469.

[Ryu et al., (2004)] Ryu, K. S., Shaikh, K., Goluch, E., Fan, Z., \& Liu, C. (2004). Micro magnetic stir-bar mixer integrated with parylene microfluidic channels. Lab on a Chip, 4(6), 608-613.

[Lin et al., (2011)] Lin, C. M., Liu, H. P., Lai, Y. S., Tseng, C. C., Chen, C. Y., \& Wo, A. M. (2011). Micromixing via recirculatory flow generated by an oscillatory microplate. Microfluidics and nanofluidics, 11(2), 167-176.

[Chen et al., 2014] Chen, C. Y., Lin, C. Y., \& Hu, Y. T. (2014). Inducing 3D vortical flow patterns with 2D asymmetric actuation of artificial cilia for high-performance active micromixing. Experiments in Fluids, 55(7), 1-9.

[Rahbar et al., 2014] Rahbar, M., Shannon, L., \& Gray, B. L. (2014). Microfluidic active mixers employing ultra-high aspect-ratio rare-earth magnetic nano-composite polymer artificial cilia. Journal of Micromechanics and Microengineering, 24(2), 025003.

[Kunti et al., 2017] Kunti, G., Bhattacharya, A., \& Chakraborty, S. (2017). Rapid mixing with high-throughput in a semi-active semi-passive micromixer. Electrophoresis, 38(9-10), 13101317.

[Erkoç et al., 2016] Erkoç, E., Fonte, C. P., Dias, M. M., Lopes, J. C. B., \& Santos, R. J. (2016). Numerical study of active mixing over a dynamic flow field in a T-jets mixer-Induction of resonance. Chemical Engineering Research and Design, 106, 74-91. 
[1] ANSYS Inc. (2011). ANSYS Fluent 14.0: User's Guide.

[Cheri et al., 2013] Cheri, S. M., Latifi, H., Salehi Moghaddam, M., \& Shahraki, H. (2013). Simulation and experimental investigation of planar micromixers with short-mixing-length. Chemical Engineering Journal, 234, 247-255.

[Hsiao et al., 2014] Hsiao, K. Y., Wu, C. Y., \& Huang, Y. T. (2014). Fluid mixing in a microchannel with longitudinal vortex generators. Chemical Engineering Journal, 235, 27-36.

[Sarkar et al., 2014] Sarkar, S., Singh, K. K., Shankar, V., \& Shenoy, K. T. (2014). Numerical simulation of mixing at 1-1 and 1-2 microfluidic junctions. Chemical Engineering and Processing: Process Intensification, 85, 227-240.

[White, 2003] White, F.M. (2003). Fluid mechanics. McGraw-Hill, New York.

[Ashraf et at., 2011] Ashraf, M. A., Young, J., \& Lai, J. C. S. (2011). Reynolds number, thickness and camber effects on flapping airfoil propulsion. Journal of Fluids and Structures, 27(2), 145-160.

[Hoke et al., 2015] Hoke, C. M., Young, J., \& Lai, J. C. S. (2015). Effects of time-varying camber deformation on flapping foil propulsion and power extraction. Journal of Fluids and Structures, $56,152-176$.

[Biancoline et al., 2014] Biancolini, M. E., Viola, I. M., \& Riotte, M. (2014). Sails trim optimisation using CFD and RBF mesh morphing. Computers \& Fluids, 93, 46-60.

[] Design Exploration User's Guide. ANSYS, Inc. Southpointe 275 Technology Drive Canonsburg, PA 15317.

[Myers et al., 2016] Myers, R. H., Montgomery, D. C., \& Anderson-Cook, C. M. (2016). Response surface methodology: process and product optimization using designed experiments. John Wiley \& Sons.

[Goldberg, 1989] Goldberg, D.E. (1989) Genetic algorithms in search, optimization and machine learning. Addison-Wesley, Reading, MA, USA.

[Deb, 2001] Deb, K. (2001) Multi-objective optimization using evolutionary algorithms. Wiley, Chichester. 
[Deb et al., 2000] Deb, K., Agrawal, S., Pratap, A., \& Meyarivan, T. (2000, September). A fast elitist non-dominated sorting genetic algorithm for multi-objective optimization: NSGA-II. In International Conference on Parallel Problem Solving From Nature (pp. 849-858). Springer Berlin Heidelberg.

[Zitzler et al., 2001] Zitzler, E., Laumanns, M., \& Thiele, L. (2001) SPEA2: Improving the performance of the Strength Pareto Evolutionary Algorithm. Technical Report No. 103, Computer Engineering and Communication Networks Laboratory (TIK), Swiss Federal Institute of Technology (ETH), Zurich, Switzerland

[Ortega-Casanova and Castillo-Sanchez, 2017] Ortega-Casanova, J., \& Castillo-Sanchez, S. I. (2017). On using axisymmetric turbulent impinging jets swirling as Burger's vortex for heat transfer applications. Single and multi-objective vortex parameters optimization. Applied Thermal Engineering, 121, 103-114.

[Chung and Shih, 2007] Chung, C. K., \& Shih, T. R. (2007). A rhombic micromixer with asymmetrical flow for enhancing mixing. Journal of Micromechanics and Microengineering, 17(12), 2495.

[Parsa et al., 2014] Parsa, M. K., Hormozi, F., \& Jafari, D. (2014). Mixing enhancement in a passive micromixer with convergent-divergent sinusoidal microchannels and different ratio of amplitude to wave length. Computers \& Fluids, 105, 82-90.

[Ortega-Casanova, 2018] Ortega-Casanova, J. (2018). CFD study about the effect of using multiple inlets on the efficiency of a micromixer. Assessment of the optimal configuration working as a microreactor. Submitted to: Chemical Engineering and Processing: Process Intensification (under review). 